\title{
El factor emocional en la búsqueda de información
}

\author{
Emotional factors in information search
}

Marta Platero Gómez, Eva Ortoll EsPinet

Universidad Oberta de Catalunya, España, \{ mplaterog | eortoll $\} @$ uoc.edu

\begin{abstract}
Resumen
El estudio de los aspectos afectivos en las Ciencias de la Información está tomando impulso en los últimos años. El objetivo de esta investigación es analizar las expresiones emotivas de los usuarios durante el proceso de búsqueda de información en un sistema de información. A partir de los modelos teóricos desarrollados por Kuhlthau $(1991,2004)$ y Nalh (2005) se escoge un estudio de caso cualitativo para indagar en el análisis de la emotividad como estrategia complementaria de evaluación de los sistemas de información. Los resultados del estudio indican que las emociones experimentadas durante la interacción pueden modificar los patrones cognitivos y las estrategias de búsqueda de los usuarios. La alteración de patrones puede explicarse según emociones lo que las convierte en indicadores sobre la eficiencia en la interacción con el sistema.
\end{abstract}

Palabras clave: Afectividad. Emociones. Paradigma afectivo. Patrones emocionales. Comportamiento informacional. Evaluación de sistemas.

\section{Introducción}

El presente artículo se enmarca en las líneas de investigación de los autores Kuhlthau (1991, 2004) y Nahl $(2005,2007)$, que analizan la afectividad y su relación con el proceso de búsqueda de la información desde una perspectiva cognitiva y emocional. Siguiendo a ambos autores, la investigación que aquí se presenta es un estudio de caso para ver cómo se desarrollan los patrones cognitivos según las emociones de los usuarios. El caso se desarrolla a partir de la interacción de los usuarios en el portal SIDBRINT de Ciencias Sociales especializado en la Guerra Civil española. SIDBRINT comenzó como proyecto de investigación I+D financiado por el Ministerio de Economía y Competitividad (2010-2013) y actualmente es un proyecto de la Universidad de Barcelona (http://sidbrint. ub.edu). El objetivo del portal es recopilar información y dar visibilidad y entidad a los brigadistas extranjeros que participaron en la Guerra Civil española. Este sistema de información se plantea como un entorno colaborativo, enfocado

\begin{abstract}
The study of affective aspects is being encouraging on Information Science in recent years. The objective of this research is to analyze the emotive expresssions performed on information seeking process during information system interaction proccess. From the theoric models developed by Kuhlthau $(1991,2004)$ and Nahl (2005) a cualitative case study is developed to research about the emotivity as complementary strategy to evaluate information systems. Preliminary results show that experimented emotions during interaction can modify the cognitive patterns and seeking strategies adopted by users. Variations over the patterns can be explained by emotions, which converts them into indicators about system interaction efficiency.
\end{abstract}

Keywords: Affectivity. Emotions. Affective paradigm. Emotional patterns. Information behavior. System evaluation.

hacia historiadores, documentalistas y profesionales de las Ciencias de la Información.

El artículo se estructura de la siguiente manera: el apartado 2 presenta una revisión de la literatura sobre afectividad y comportamiento informacional, en el apartado 3 se explica el marco teórico a partir del cual se plantea el estudio. El apartado 4 presenta los aspectos metodológicos y de visualización de resultados. El apartado 5 analiza los resultados obtenidos. En el apartado 6 se discuten dichos resultados en relación a los marcos teóricos utilizados. Finalmente el apartado 7 presenta las principales conclusiones del estudio de caso.

\section{Revisión de la literatura}

Las emociones son parte esencial de la actividad humana, incluida la búsqueda de información. Los elementos afectivos y emocionales no son ajenos a los procesos cognitivos de las personas y ejercen una función regulatoria entre cuerpo y cerebro con repercusiones en la toma racional de decisiones y en la percepción (Lopa- 
tovska y Arapakis, 2011). La presencia de dichos aspectos en la agenda de los profesionales de la información la encontramos en autores como Wilson (1981, 2006), Kuhlthau (1991, 2004), Nahl (2007), Chatman (1991) o Dervin (1992) que han contribuido al desarrollo de áreas de investigación relacionadas con estados afectivos y el proceso de dotación de significado (sense-making) o de la afectividad combinada con la interacción usuario ordenador, lo que se ha denominado affective $\mathrm{HCl}$ o affective computing (Picard, 1997, 2003).

Recientemente ha resurgido el interés por contemplar la afectividad en los estudios del ámbito de las Ciencias de la Información y la Documentación. Una muestra de ello la encontramos en el número especial del American Society for Information and Science and Technology (ASIS\&T) Information and emotion: the emergent affective paradigm in information behavior research and theory, editado por Nahl y Bilal (2007). Por su parte, Fouire y Julien (2014a) abogan por concienciar a los académicos sobre estos aspectos y realizan una revisión bibliográfica en la que proponen una agenda de investigación a partir de la cual abordar las experiencias emocionales en la interacción usuariosinformación. Los mismos autores sugieren la necesidad de instaurar el paradigma afectivo en el currículum de las Ciencias de la Información y la Documentación (Fouire y Julien, 2014b). En la misma línea de pensamiento Lopatovska y Arapakis (2011) revisan trabajos sobre la afectividad en tres áreas muy concretas: Biblioteconomía y Ciencias de la Información, Recuperación de Información e Interacción de Humanos con Ordenadores.

La investigación sobre el comportamiento informacional, y de manera más específica el proceso de búsqueda (information-seeking behaviour) y la interacción con los sistemas de recuperación, se ha enfocado tradicionalmente sobre los aspectos cognitivos del proceso o de usabilidad del sistema, sin prestar demasiada atención a los factores emocionales que lo circundan (Hudlicka, 2003), exceptuando trabajos anteriormente mencionados como los de Wilson, Kuhlthau, Nahl o Dervin.

Así, después de la revolución cognitivista de los años 60 varios autores comienzan a interesarse por los elementos emotivos que afectan al proceso de búsqueda de información. El ser humano responde a multitud de estímulos: el entorno, el contexto de una situación o las tareas que desempeña. Muchos de estos estímulos provocan respuestas en el ámbito afectivo (contexto agradable/desagradable) y en el ámbito físico (desempeña una tarea porque así lo deci- de) que no se explican solo con factores cognitivos. Bandura (1989) desarrolla la teoría de la cognición social para explicar este fenómeno. Las influencias cognitivas, comportamentales y del entorno se relacionan entre sí de manera determinante. Aunque no se explicitan las emociones se acepta que el entorno y las reacciones que causan en el usuario juegan un papel al nivel del proceso cognitivo.

En este contexto cada persona procesa una serie de estímulos subjetivos individuales, tanto cognitivos, como afectivos y físicos (Savolainen, 2015; Fourie y Julien 2014). Diversos autores han analizado la interrelación de estas facetas en el individuo para comprender el proceso de búsqueda de información (Fidel, 2012; Savolainen, 1995; Lopatovska y Arapakis, 2011; Kuhlthau, 2004; Nahl, 2007). En 1992, Dervin describe el sense-making (Dervin, 1992) como un proceso a través del cual se otorga significación a la información recibida. El usuario debe percibir una brecha informativa que lo impulse a realizar búsqueda(s) para solventar su necesidad. Los factores cognitivos (percibir una necesidad) se conjugan con los afectivos (solventar la necesidad) y los físicos (ejecutar la búsqueda). No obstante, no hay una interrelación clara entre los tres ámbitos, algo que también sucede en otros modelos, como el Everyday Life information Seeking (ELIS) de Savolainen (1995).

Las investigaciones precedentes (Dervin, 1992; Kuhlthau, 1991; Picard, 1997) muestran como la afectividad y las acciones intervienen en el proceso de búsqueda de información en medida similar a los factores cognitivos. Estos factores han sido objeto de estudio de muchas disciplinas (psicología, medicina, biología, filosofía) lo que facilita su identificación y proporciona a los investigadores diversos métodos y escalas para abordarlos. En el caso de las emociones, la subjetividad inherente a ellas las convierte en elementos de difícil estudio. Aunque puedan reconocerse (alegría, felicidad, tristeza, rabia...) no son fácilmente cuantificables. Además, se desarrollan dentro del individuo como una experiencia subjetiva, lo que dificulta su apreciación. Dos individuos que experimenten una emoción similar, pueden hacerlo en grados diferentes y con respuestas y reacciones contrapuestas, ya que la respuesta emocional depende del contexto, de la situación, de las vivencias del individuo y del momento en que suceda (Case, 2007).

El análisis de las emociones y la búsqueda de información suele estar representado por estudios que se centran en las emociones negativas (Lazar, Jones, y Shneiderman, 2006) o en las positivas (Fulton, 2009). La relación entre la afectividad y la cognición está menos explorada 
aunque hay estudios que abordan el tema, como Kuhlthau (1991, 1999, 2004), Nahl (2005a, 2005) o Savolainen (2015).

Desde un punto de vista metodológico y de recogida de datos, Lopatovska y Arapakis (2011) identifican distintas metodologías y enfoques para analizar las experiencias emocionales de los individuos. Desde un punto de vista teórico los modelos de Kuhlthau (1991, 2004) y Nahl (2007) permiten interrelacionar los factores emotivos y cognitivos, y trazar un patrón general afectivo-cognitivo. Estos dos últimos modelos se consideran los más completos para estudiar los elementos emocionales en el proceso de búsqueda de información (Fourie y Julien 2014a, 2014b; Savolainen 2015).

\section{Marco teórico}

Partiendo de los marcos conceptuales de Kuhlthau y Nahl, el presente estudio pretende testear la utilidad de dichos modelos para aproximarse a los patrones emocionales de interacción como estrategia para evaluar un sistema de información. La pregunta de investigación que se plantea es: ¿Qué expresiones emocionales manifestadas por los usuarios están influyendo en la efectividad de las búsquedas en un sistema de información?

El modelo de Kuhlthau analiza el comportamiento informacional durante el proceso de búsqueda de información con la incertidumbre como eje vertebrador. El "Information Searching Process" (ISP) de Kuhlthau $(1991,2004)$ parte del estudio de la incerteza y ansiedad en el usuario como motivadores de la búsqueda. Parte de su fortaleza es la relación contextual con las tareas desarrolladas por los usuarios, que permite precisar los factores cognitivos y afectivos (Savolainen, 2014). La búsqueda de información se identifica con un proceso constructivo en que el usuario atraviesa una serie de subprocesos para alcanzar (o no) un estado afectivo (satisfacción/disgusto) por medio de información que resuelva (o no) una necesidad cognitiva a través de una búsqueda pertinente (acción).

El modelo se divide en siete etapas según los estados afectivos del proceso de búsqueda (Kuhlthau, 1991, 2004). Cada una de ellas relaciona los tres campos de la experiencia humana (afectivo, cognitivo y físico). Las divisiones se establecen basándose en los procesos cognitivos y afectivos según una perspectiva constructivista y holística. Las acciones son las que marcan los estados del usuario y determinan el porqué de los estados cognitivos: el usuario siente, en consecuencia analiza y por último actúa. El proceso de búsqueda no sigue pautas ordenadas y claras sino que se trata de un proceso complejo en el que la incertidumbre y la ansiedad impulsan la búsqueda y explican la interacción usuario-sistema. El uso de este modelo en el presente estudio es parcial, ya que los usuarios interaccionan con un sistema que ya les ofrece el tema y los recursos de información. En este estudio y según el modelo de Kuhlthau, se posicionan en la etapa de exploración. Es a partir de este momento en que el modelo se centra en los aspectos cognitivos y afectivos implicados en la interacción con el sistema de búsqueda.

Nahl (2007) por su parte aborda el comportamiento informacional desde una visión holística y construccionista conjugando aspectos tecnológicos, estructuras sociales y biología humana.

El modelo Socio-Biological Information Technology Model (SBIT) de Nahl (2007) establece como eje central el proceso de adaptación que experimentan los usuarios ante el entorno informativo, abordando la afectividad desde la perspectiva biológica del ser humano. La relación entre el comportamiento informacional y el sistema afectivo se establece a través de dos funciones biológicas: la recepción afectiva de información y su uso afectivo en un proceso dinámico de recepción y uso. Según el sistema biológico que haya entrado en juego para procesar la información, Nahl los clasifica en tres etapas: sensorimotor (percibir información), cognitivo (procesar y valorar información) y afectivo (evaluar y consumir la información percibida).

Nahl explica cómo la tecnología permite la satisfacción de las affordances, es decir, la satisfacción de aquellas acciones que el objeto (en este caso el sistema) por el hecho de serlo, le sugieren al usuario que debe realizarlas para utilizarlo. Estas acciones desencadenan una serie de estímulos que afectan primero al sistema sensorimotor, al cognitivo y por último al afectivo, que es el que evalúa la información recibida. A partir de aquí el usuario puede hacer un uso de dicha información, invirtiendo el flujo de funcionamiento de los sistemas: en primer lugar está la intención del usuario a utilizar esa información (motivación, sistema afectivo), después la planificación sobre el uso de la información (sistema cognitivo) y por último la representación que hace el usuario con esa información (sensorimotor) y que es la responsable de la estrategia de respuesta. Esta clase de interacción es lo que se denomina optimal affordances, y su diseño debe permitir que el usuario influya sobre el sistema para modificar su respuesta. El modelo de Nahl analiza en la interacción con el sistema 
de manera integral por lo que se adecúa mejor a los propósitos de este estudio. Como marco referencial metodológico es más completo, permitiendo una total aplicación del mismo.

Los factores afectivos y cognitivos se retroalimentan mutuamente. Dado el ciclo de desarrollo del SBIT, la cognición se sugiere como dependiente de la afectividad (Savolainen, 2014). En este caso, son las emociones las que van a definir el curso del proceso cognitivo, y por ende, de las acciones.

La secuencia entre cognición y afectividad, desde que se percibe la información hasta que se responde es sensorimotor - cognición - afectividad - afectividad - cognición - sensorimotor, siendo la respuesta cognitiva planificada según la afectividad desarrollada por el usuario. El proceso cognitivo se delimita parcialmente en función de la emoción experimentada. Savolainen (2014, p. 190) extrae esta misma conclusión al analizar la interrelación entre ambos factores en este modelo.

La interacción entre usuario y sistema queda reflejada en el gráfico anterior (Figura 1), en dónde se evidencia la relación recíproca entre usuario y sistema, aunque es la estructura del sistema la que termina segmentando a los usuarios por perfiles. La interacción del sistema con los discursos cognitivos y la afectividad derivan en los patrones emocionales de cada individuo. La reciprocidad entre el sistema y la cognición y afectividad todavía no existe, si bien se está avanzando en que el sistema pueda reconocer estas características. En el esquema, esta nueva línea de investigación se marca con una flecha discontinua.

El desarrollo de acciones del usuario sirve para inferir los procesos afectivos y cognitivos atendiendo a la secuencia de sistemas descrita en el modelo de Nahl: sistema sensorimotor - sistema cognitivo - sistema afectivo - afectividad cognición - acción. El usuario percibe la información (sensorimotor), la convierte en una señal procesable (cognitivo) y finalmente responde emocionalmente (afectivo). La respuesta se basa en esa emoción (afectivo) interpretándola (cognitivo) para decidir cómo actuar, y ejecutando (sensorimotor) una acción en respuesta. Se sugiere la delimitación parcial del proceso cognitivo en función de la emoción desarrollada, una idea también explorada por Savolainen en su revisión del modelo de Nahl (Savolainen 2014, p. 190).

La clasificación emocional se efectúa atendiendo a un modelo dual de valencias (positivo/negativo).Varios autores utilizan este tipo de clasificación de emociones (Pluthchick, 1980;
Thayer, 1996; Scherer, 2005). Thayer y Scherer incluyen en sus modelos la activación emocional activo/pasivo (Lopatovska, Arapakis, 2011).

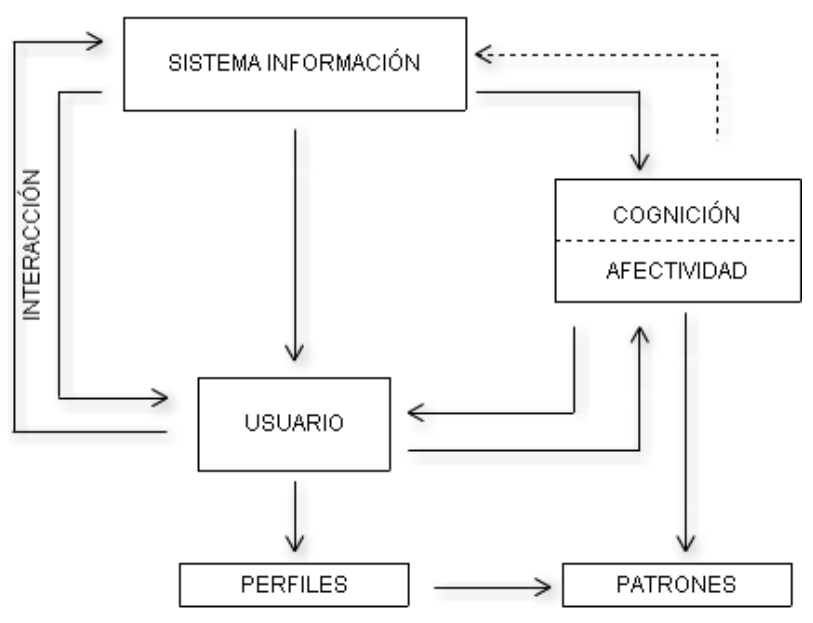

Figura 1. Diagrama sobre el proceso de análisis y representación de los patrones emocionales

Partiendo de dichos marcos se designan tres grupos de emociones: positivas, negativas y neutras. Las positivas se representan con tonos rojos, las negativas con tonos azules y las neutras con tonos verdes. La intensidad del tono indica la activación emocional: a mayor intensidad mayor activación y mayor valencia. Las emociones con la gama verde tienen una valencia neutra, en los límites entre emoción positiva y negativa mientras que su activación puede ser alta o baja.

\begin{tabular}{lll}
\hline Positivas Neutras & Negativas
\end{tabular}

Tabla I. Categorías emocionales

\section{Metodología: estudio de caso}

En este apartado presentamos los métodos utilizados para recoger y analizar los datos. El objeto de investigación es identificar los patrones afectivos que influyen en las búsquedas de información.

Para responder a la pregunta de investigación planteada previamente seleccionamos métodos de recogida de datos que identifiquen las emociones relacionadas con el uso de un sistema de información. Fidel (2012) aboga por los estudios de caso contextuales para abordar la complejidad del comportamiento informacional. En este caso se desarrolla un estudio de caso en con- 
texto, sobre un sistema de información concreto -Portal SIDBRINT- dado que la interrelación acción-afectividad-cognición debe analizarse en el contexto en el que se desarrolla el comportamiento (Case, 2002).

Lopatovska y Arapakis (2011) identifican tres métodos para recoger las experiencias emocionales de los individuos: procesamiento de señales neuro-psicológicas; observación y autoinforme. En nuestro caso empleamos una combinación de observación y auto-informe.

La muestra utilizada es intencionada o de conveniencia (Wildemuth, 2009) en la que participan 12 usuarios. La muestra identifica los segmentos del target del portal: historiadores y expertos en Guerra Civil (7 usuarios); documentalistas especializados en sistemas de información (5 usuarios), habiendo participado estos últimos en el desarrollo conceptual del portal. La muestra es apropiada en el contexto de un estudio cualitativo que no pretende hallar generalizaciones sino obtener nuevo conocimiento para diseñar un estudio más amplio. La separación en dos grupos se explica por los conocimientos previos de los usuarios: los historiadores y expertos tienen aptitudes suficientes para desarrollar búsquedas propias según sus intereses. Por otro lado, los documentalistas expertos en sistemas de información no tienen conocimiento temático suficiente para llevar a cabo búsquedas propias. Por ello, se les facilitan las tareas básicas en torno a las cuales se planteó el diseño del sistema (Prades, 2008). A este último grupo (documentalistas) se le presupone una ventaja en cuanto a la interacción con el portal.

\subsection{Recogida de datos}

Se recogen los datos mediante grabaciones con Camtasia 8, un software que permite realizar grabaciones de la interacción usuario-sistema en tiempo real. Además se toman notas en campo en cada sesión de interacción usuariosistema. Estos registros permiten contrastar las interacciones en el sistema con el lenguaje corporal y la expresión facial. Siguiendo el método del Think Aloud (Wildemuth, 2009) a cada usuario se le pide que comente en voz alta lo que piensan durante la interacción. Esto facilita el seguimiento del proceso cognitivo y emocional y comprender el razonamiento de los usuarios durante la interacción: el discurso se modifica según la interacción, lo que permite comprender la selección de estrategias adoptadas en tiempo real. Las grabaciones tienen una duración entre 10 y 42 minutos. Las sesiones de interacción se dividen en dos grupos: (a) Interacciones libres: En este grupo se hallan los usuarios (7) conocedores del tema (Guerra Civil). Este conocimiento previo permite que interaccionen con el portal de manera libre, dado que cuentan con recursos propios (estudios previos) para realizar búsquedas. No hay tareas predeterminadas. Los usuarios crean un discurso cognitivo propio. La falta de tareas predeterminadas permite evaluar la manera de búsqueda de los usuarios que estudian este tema, siendo público objetivo del portal.

(b) Interacciones guiadas: En este grupo se hallan los usuarios (5) expertos en sistemas de información, que colaboran en el diseño conceptual del portal y que no son expertos en la Guerra Civil. Los expertos en sistemas de información sirven para calibrar la eficiencia de los sistemas de clasificación que se integran en el portal diseñado por otros expertos en sistemas digitales. Dado que no tienen conocimientos específicos sobre la Guerra Civil española y los brigadistas, se les facilitan una serie de tareas predeterminadas, las que se emplearon como base para diseñar el prototipo del portal (Prades, 2008). Los usuarios deben amoldarse a un guión que modela su discurso cognitivo.

Las interacciones libres terminan cuando los usuarios ya no son capaces de plantearse más escenarios de interés. Las interacciones guiadas finalizan cuando se terminan las sub-tareas, sean satisfactorias o no.

\subsection{Procedimiento de análisis y visualización}

Una vez efectuadas las grabaciones, se transcriben para su análisis. Éste se efectúa siguiendo las pautas establecidas por Scherer (2005). La codificación de estos fragmentos se realiza en base a las locuciones de los usuarios, con comentarios sobre los gestos faciales y lenguaje corporal de los usuarios que contribuyan a identificar la emoción correspondiente.

La representación de los patrones se realiza asociando cada emoción con la interacción que la desencadena. Esta interacción usuariosistema se clasifica según tres tipos de acciones (Tabla II).

\begin{tabular}{ll}
\hline Click & $\begin{array}{l}\text { Pulsación del ratón en una zona } \\
\text { del sistema }\end{array}$ \\
\hline Scroll & Subir y bajar la interfaz del sistema \\
\hline Tecleo & $\begin{array}{l}\text { Usar el teclado para interactuar } \\
\text { con el sistema }\end{array}$ \\
\hline
\end{tabular}

Tabla II. Acciones 
Las emociones de los usuarios se representan gráficamente en patrones clasificados por colores. (Figura 2) Cada emoción se ubica de manera secuencial.

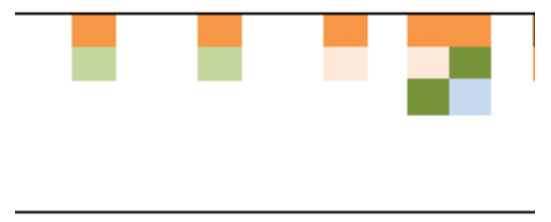

Figura 2. Fragmento de patrón emocional-unidad de medida del patrón.

Esta asociación emoción-acción permite utilizar la acción como unidad de medida del patrón, representándose en el gráfico con un espacio cuadrado. Cada uno de los espacios se colorea según la acción que represente, o bien queda en blanco si la acción no tiene emoción asociada. El largo del patrón queda determinado por la cantidad de acciones realizadas en la interacción, mientras que el largo queda determinado por la emoción o emociones que desencadenan una misma acción. No hay una relación proporcional entre el tiempo de interacción y la cantidad de acciones, varía según el comportamiento de cada usuario.

\section{Resultados}

La presentación de resultados se estructura según los dos perfiles identificados. Se pretende descubrir en qué medida las emociones influyen en los procesos cognitivos del proceso de búsqueda de información y sí existen diferencias entre perfiles.
Atendiendo a la codificación de las transcripciones y el análisis de las grabaciones se codifican 17 estados emocionales que se utilizan como elemento para perfilar los patrones (Tabla III).

\begin{tabular}{lll}
\hline Positivos & Neutros & Negativos \\
\hline Alegría & Sorpresa & Enfado \\
\hline Ganas & Duda & Indignación \\
\hline Interés & Incredulidad \\
\hline Satisfacción & Frustración \\
\hline Comprensión & Nervios \\
\hline & Molestia \\
\hline & Inseguridad \\
\hline & Decepción \\
\hline & Aburrimiento \\
\hline
\end{tabular}

Tabla III. Emociones

La identificación de las emociones se hace según una leyenda de colores que permita descifrar el desarrollo del patrón emocional (Figura 3).

5.1. Interacciones libres: usuarios expertos en el tema del portal

Estos usuarios realizan interacciones libres y muestran patrones más positivos (en relación a la búsqueda) que los usuarios del otro perfil. Sin embargo sus picos de negatividad muestran valencias más altas. Los picos de negatividad se relacionan principalmente con:

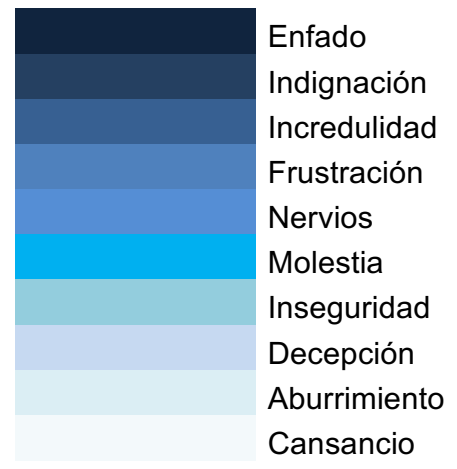

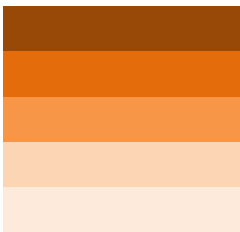

\section{Regocijo/Alegría}

Ganas

Interés

Satisfacción

Comprensión
Sorpresa

Duda

Figura 3. Leyenda de emociones por colores

- Falta de comprensión de la interfaz: -"iLo que me sale siempre son libros!" - "No me ha encontrado nada?" Y... “¿y dónde sale que no me ha encontrado nada?" No obtener los resultados esperados según en el conocimiento de los usuarios: "No, tampoc (el usuario da un puñetazo en la mesa)." "Ostra, esto es..." El usuario no es capaz de formular 
una búsqueda válida: "Hmmm (ruído imitando a un sollozo)." Problemas con la visualización de resultados: "Y aquí te pro... te pro...mmm te permite poneeeer el mes, pero ¿el año?."

- No comprender el sistema de etiquetado: “¿No están entrados por países?” “EÉsto es la cerca avançada?" Aburrimiento y falta de interés: "Entro aquí... esto de arriba no he entendido nada ¿eh?"

Los usuarios acceden al sistema para recopilar información, pero el sistema no les facilita pistas sobre cómo hacerlo, obligándolos a plantearse nuevos métodos. Se observan algunos problemas en el diseño del portal, como deficiencias al mostrar los mensajes del sistema, mal funcionamiento de algunas etiquetas interactivas 0 títulos de apartados poco descriptivos que confunden a los usuarios.

\subsection{Interacciones guiadas - Usuarios expertos} en sistemas de información

Estos usuarios han colaborado con el desarrollo del proyecto y conocen la metodología de clasificación del portal. En sus patrones emocionales predominan las valencias bajas para las emociones positivas y negativas. Esto implica una pasividad del usuario en las interacciones, lo que puede traducirse como falta de implicación. Las emociones positivas de alta valencia se relacionan con el interés de los usuarios: dos de ellos en momentos concretos de la interacción deciden realizar búsquedas alternativas para comprobar si las búsquedas directas y por navegación llegan a iguales resultados. Los picos de negatividad registrados se deben a:

a) Falta de comprensión sobre cómo interactuar con la interfaz del sistema: "No tengo que clicar... es que no me acuerdo de nada."

b) No obtener resultados en las consultas: "Cero items. Pues... pues no sé cómo hacerlo,"

c) El usuario no es capaz de formular la búsqueda a nivel conceptual: "Bueno es igual. No lo sé hacer."

Los usuarios guiados muestran un patrón con pocas emociones y bajas valencias a pesar de conocer el sistema. El tener un listado de búsquedas les ahorra hacer un esfuerzo cognitivo más allá de utilizar la búsqueda directa para llegar a los resultados. La búsqueda que todos fallan es una por navegación en la que el sistema no facilitaba el enlace correspondiente. Para resolverla los usuarios tenían que realizar una búsqueda directa. Dos de ellos no lo intentan, dando la búsqueda por fallada. Los demás usuarios sí lo hacen, aunque sin investigar los resultados obtenidos. Esto indica que no hay un interés de los usuarios sobre la tareas, sino que su finalidad es exclusivamente resolverlas sin profundizar. El comportamiento muestra que ante una serie de búsquedas predeterminadas los usuarios limitan su esfuerzo cognitivo.

\subsection{Comparativa de patrones}

Los patrones de ambos perfiles de usuarios difieren tanto en intensidad como en frecuencia de las emociones desencadenadas. Por una parte, los usuarios con conocimientos en Guerra Civil experimentan emociones más fuertes y la relación cognición-afectividad es más marcada. Los usuarios expertos en sistemas no obtienen una ventaja sustancias para realizar las búsquedas, y sus patrones emocionales son más planos y con un número de emociones menor.

Los usuarios expertos en Guerra Civil deben generar su propio discurso cognitivo para interrogar al sistema. Sus conocimientos sobre la temática les permiten formular consultas, pero el desconocimiento del sistema dificulta la interacción. Generar un discurso cognitivo propio hace que los usuarios se impliquen más con el sistema, probando diversas combinaciones para llegar al resultado y experimentando emociones positivas al conseguirlo. La contrapartida es experimentar emociones negativas cuando los resultados no son los esperados o la interacción con el sistema no es adecuada (Figura 4). Los usuarios guiados y expertos en sistemas de información no llegan a desarrollar un proceso cognitivo propio, sino que se centran en obtener resultados para satisfacer las tareas que se les proponen. Las valencias de sus emociones son bajas y sus patrones emocionales son más planos que los de los usuarios no guiados (Figura 5). Si bien el conocimiento del sistema hace que los usuarios reduzcan sus emociones negativas, también se aplica a las positivas. Los usuarios no guiados y expertos en Guerra Civil experimentan una variedad emocional más amplia, tanto de emociones positivas como negativas (Figura 4), mientras que los usuarios guiados experimentan una paleta emocional más reducida (Figura 5).

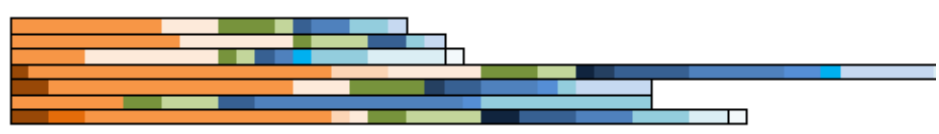

Figura 4. Acumulativo de emociones usuarios no guiados 


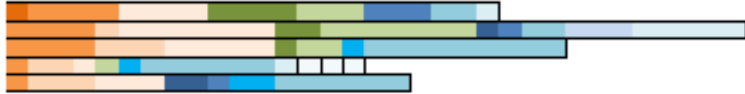

Figura 5. Acumulativo de emociones usuario guiados
La frecuencia emocional también difiere bastante entre los usuarios no guiados y los guiados. Los primeros experimentan muchas más emociones en su sesión de interacción que los usuarios guiados.
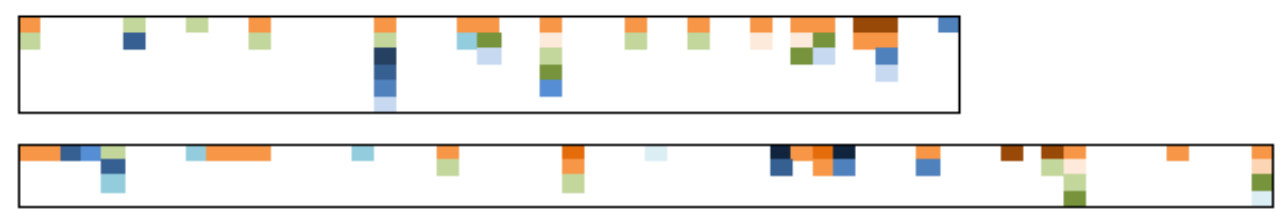

Figura 6. Gráficos emocionales de dos usuarios no guiados

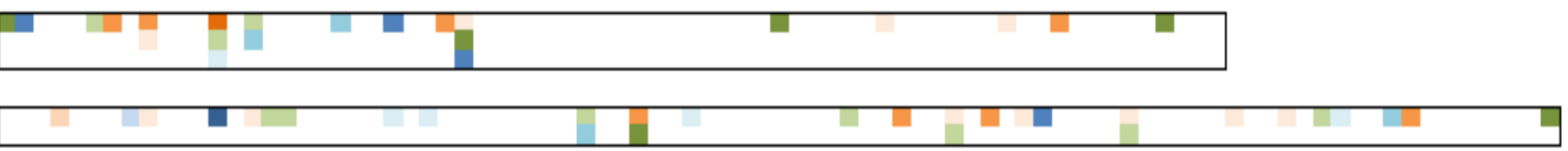

Figura7. Gráficos emocionales de dos usuarios guiados

Identificar los momentos más positivos y negativos permite rastrear los aspectos en que la comunicación entre el sistema y el usuario no se desarrollan correctamente. Ocho de los doce usuarios experimentan emociones de alta valencia, tanto positiva como negativa, que se producen como respuesta a una acción.

Tras el estudio se aprecian indicios de que los usuarios con conocimientos sobre el tema tratado en el portal experimentan emociones más fuertes y se implican más en las búsquedas. Los usuarios expertos en sistemas de información y sin conocimientos específicos desarrollan una vinculación menor y un patrón emocional más plano. Sus conocimientos en sistemas no les facilitan la búsqueda en comparación con los otros usuarios, y el hecho de facilitarles tareas predeterminadas induce a un menor esfuerzo cognitivo que se traduce en menor emotividad. Se confirma la relación afectividad-cognición que determina los patrones de búsqueda y las estrategias.

La falta de un mensaje que indique que el proceso de búsqueda ha finalizado empobrece la interacción con el sistema: los usuarios no reconocen cuándo se termina la tarea, especialmente cuando no hay resultados. La frustración y desánimo resultantes desalientan a los usuarios, evitando que investiguen a través del sistema. En cuanto a la presentación de las fichas, varios usuarios confunden la pre-visualización de registros (con escasa información) con el registro completo, evitando que cliquen sobre la pre-visualización para acceder al contenido completo, y causando la impresión de que están ante un sistema mal documentado.

\section{Discusión}

Los patrones emocionales facilitan el análisis de los planos cognitivo y afectivo en la búsqueda de información. La revisión de secuencias emocionales sugiere que los procesos cognitivos se alteran por falta de comunicación usuariosistema. Esta idea se refuerza por otros estudios sobre affective computing (Picard, 1997, 2003; Hudlicka, 2003; Bamidis et al. 2004). La relación entre afectividad y cognición se perfila como dependiente: los usuarios con búsquedas programadas realizan un esfuerzo cognitivo menor que aquellos que no las tienen. El reflejo emocional en los patrones es muy claro: la falta de discurso cognitivo disminuye la cantidad de emociones experimentadas. Un análisis a micronivel siguiendo a Nahl sugiere que esta falta de discurso dificulta la percepción y valoración de la información, modificando las respuestas afectivas. Las modificaciones afectan a las respuestas físicas de los usuarios, y así sucesivamente, explicando los cambios cognitivos.

El conocimiento previo de algunos usuarios les permite saltarse las etapas iniciales del proceso de búsqueda identificadas por Khulthau. Sin embargo, su conocimiento no responde a los parámetros del sistema, no hay una interacción usuario-sistema adecuada, afectando a la comprensión y obligando a los usuarios a retroceder en su secuencia cognitiva. La arquitectura del sistema denota falta de correlación entre lo que el usuario espera encontrar y lo que encuentra. Deben replantearse las estrategias de búsqueda alterando los términos de búsqueda (formulación), combinando búsqueda directa y contex- 
tual (selección) o recurriendo al paginador para ver resultados (exploración) sin una secuencia clara. Siguiendo a Kuhlthau los usuarios expertos en Guerra Civil se enfrentan al sistema desde la etapa de recolección (collection) pero la imposibilidad de comunicarse con él, hace que retrocedan en la secuencia cognitiva.

El acumulativo de emociones corrobora este análisis: (a) los usuarios expertos en guerra Civil acumulan más estados de duda y frustración que según Khulthau se corresponden con la faceta de exploración, y además tienen emociones más polarizadas. Experimentan un retroceso en el proceso cognitivo por una comunicación deficiente que fomenta sus emociones negativas, reconduciendo negativamente sus pautas cognitivas. (b) Los usuarios expertos en sistemas de información no experimentan una variación sustancial de sus emociones en la recuperación de información. Lopatovska (2009) lleva a cabo un estudio que confirma esta idea:

[...] when information was hard to find, participants did not report high frustration levels; when participants found results, they did not indicate an extreme happiness, etc.

Estos usuarios se encuentran ante un doble problema: no pueden utilizar la búsqueda directa porque no tienen conocimiento suficiente, y al no interactuar correctamente con el sistema de clasificación no son capaces de aplicar la búsqueda por navegación. El resultado es el refuerzo de emociones negativas ante la imposibilidad de conseguir resultados.

Los usuarios con un guión pautado y conocimientos sobre el sistema no muestran una mejora significativa en los resultados, su interés se centra en buscar y encontrar registros acordes a las tareas programadas, pero ni investigan ni se sienten implicados. En cuanto al proceso de búsqueda, el conocimiento previo sobre el sistema de información no supone una ventaja: su comportamiento apenas difiere del de los demás usuarios. La conclusión es que el conocimiento previo del tema permite una adecuada interrelación afectividad-cognición que facilita el uso del entorno.

Analizando los resultados según Nahl, el paso de la percepción cognitiva a la evaluación afectiva es el momento de interrelación y retroalimentación entre ambos procesos: tras la evaluación afectiva el usuario desarrolla una estrategia de asimilación informativa, una idea también explorada por Fulton (2009). Cada proceso cognitivo se evalúa afectivamente teniendo en cuenta el propio proceso y el acumulativo emocional previo. El usuario que concatena interacciones negativas acumula esta valencia que aumenta con cada nueva interacción.
Analizando los resultados según Kuhlthau, la búsqueda como proceso constructivo se identifica con claridad en el caso de que haya un patrón cognitivo que guíe las búsquedas. En el caso contrario no se aprecian signos de implicación y la interacción a nivel intelectual y emocional se desarrolla de manera plana. No hay una sucesión perceptible de etapas en el proceso de búsqueda cuando el hilo conductor a nivel intelectual no se desarrolla, dando lugar a un patrón con pocas emociones y de baja intensidad, y retrasando el desarrollo del patrón cognitivo.

A tenor de este análisis, se aprecia que los usuarios con patrones cognitivos propios (expertos) (interacciones libres) a pesar de no conocer el entorno son capaces de obtener búsquedas con resultados más eficientes, planteándose búsquedas relacionadas.

\section{Conclusiones}

Las emociones pueden modificar la búsqueda de información y alterar la eficiencia en la interacción usuario-sistema. Los picos emocionales permiten establecer los aspectos que más influyen en los usuarios, como ejecutar una búsqueda o durante el scroll para localizar un dato concreto. Estos repuntes afectivos coinciden con el cierre de un proceso cognitivo, cuando el usuario recibe información. Los repuntes negativos coinciden específicamente con tres clases de interrupción en la interacción sistema-usuario: (I) no hay resultados en el sistema (II); el sistema no responde a la interacción (III); la visualización de resultados desorienta al usuario, que recurre al retroceso para volver a puntos reconocibles de la búsqueda.

Los errores en la interacción con el sistema se producen tanto con usuarios expertos en el tema como con usuarios expertos en el sistema, lo que implica que el conocimiento previo de un sistema de información no supone una ventaja apreciable en la navegación. En cambio el conocimiento previo sobre el tema sí demuestra ser un aspecto clave para realizar búsquedas más eficientes y motivadas. Pese a esto, es necesaria una investigación en mayor profundidad para delimitar indicadores que permitan cuantificar la efectividad de un sistema de información según los patrones afectivos.

Sin embargo, es posible decir que tal como dice Nahl, afectividad y emoción se retroalimentan: los patrones cognitivos varían según las emociones, pero al mismo tiempo dichas emociones alteran el grado de implicación afectando a la cognición. En base a los modelos, el de Nahl se perfila como más acorde, mientras que el de Kuhlthau, se perfila como más limitado. La ges- 
tión de las emociones de este último las describe como consecuencia de la cognición, no como un elemento en paralelo. Por tanto aunque se trata de un modelo en profundidad su análisis se centra en una relación cognitivo-afectiva unidireccional, mientras que Nahl realiza un análisis bidireccional que se acomoda mejor a las nuevas líneas de investigación sobre la afectividad en sistemas de información.

Este estudio propone una metodología de análisis que facilita la cuantificación y representación de las emociones. El uso de patrones se convierte en un instrumento que facilita la comparación afectiva entre los dos grupos de usuarios. Esta visualización permite alcanzar una visión global y se postula como una metodología específica para el estudio de la afectividad, no sólo para este estudio de caso, sino que es extrapolable a otras investigaciones similares.

Tal como sugieren Savolainen (2015) y Fourie y Julien (2014 a, b) es necesario ahondar en estudios de caso que se aproximen al análisis de las emociones en los procesos de búsqueda de información. A pesar de que los estudios de caso no son comparables, la aplicación de metodologías similares puede ayudar en la identificación de pautas, abriendo nuevas vías de investigación.

\section{Referencias}

Bamidis, P. D., Papadelis, C., Kourtidou-Papadeli, C., Pappas, C., Vivas, A. B. (2004). Affective computing in the era of contemporary neurophysiology and health informatics. // Interacting with Computers, Vol 16:4, 715-721.

Bandura, A. (1986). Social Foundations of Thought and Action: A Social Cognitive Theory. Englewood Cliffs, NJ: Prentice-Hall.

Case, Donald O. (2007). Looking for Information. A survey of research on information seeking, needs, and behavior. $2^{a}$ Edición.Wagon Lane, Bingley: Emerald Group Publishing Limited.

Dervin, B. (1992). From the mind's eye of the user: the sense-making qualitative-quantitative methodology. // Glazier, J. D., Powell, R. R. Qualitative Research in Information Management. Englewood, CO: Libraries Unlimited. 61-84.

Fidel, Raya (2012). Human Information Interaction. An Ecological approach to Information Behavior. Cambridge, MA: The MIT Press.

Fourie, I., Julien, H. (2014). IRS, information services and LIS research: a reminder about affect and the affective paradigm... and a question. // Library Hi Tech. 32:1, 190-201.

Fourie, I. \& Julien H. (2014a). Ending the dance: a research agenda for affect and emotion in studies of information behaviour // Proceedings of ISIC, the Information Behaviour Conference, Leeds, 2-5 September, 2014. http://ln formationR.net/ir/19-4/isic/isic09.html (2015-07-28).

Fourie, I.; Julien, H. (2014b). IRS, Information services and LIS research: a reminder about affect and the affective paradigm... and a question. // Library $\mathrm{Hi}$ Tech. 32:81, 190-201.
Fulton, C. (2009). The pleasure principle: the power of positive affect in information seeking. // Aslib Proceedings. 61:3, 245-261.

Hudlicka, E. (2003). To feel or not to feel: The role of affect in human-computer interaction. // International Journal of Human Computer Studies, Vol 59, pp 1-32.

Kuhlthau, C. C. (1991). Inside the Search Process: Information Seeking from the User's Perspective. // Journal of the American Society for Information Science. 42:5 pp. 361- 371.

Kuhlthau, C. C. (2004). Seeking meaning. A process approach to library and information services. $2^{\mathrm{a}}$ Edición. Westport, Connecticut: Libraries Unlimited.

Lazar, J., Jones, A., Shneiderman, B. (2006) Workplace user frustration with computers: an exploratory investigation of the causes and severity // Behaviour and Information Technology. 25:3, 239-251.

Lopatovska, I.; Arapakis, I. (2011). Theories, methods and current research on emotions in library and information science, information retrieval and human-computer interaction. // Information Processing and Management. 47:4, 575-592.

Lopatovska, I. Searching for good mood: Examining relationships between search task and mood. // Proceedings of the American Society for Information Science and Technology. 46:1, 1-13.

Nahl, D. (2007). The centrality of the affective in information behavior. // Nahl, D.; Bilal,D. (eds). Information and emotion. The Emergent Affective Paradigm in Information Behavior Research and Theory, Medford, NJ: Information Today Inc.

Picard, R. W. (1997). Affective computing. Cambridge, MA, USA: MIT Press.

Picard, R. W. (2003). Affective computing: Challenges. /I International Journal of Human Computer Studies. 59, 55-64.

Plutchik, R. (1980). A general psychoevolutionary theory of emotion. Emotion: Theory, research and experience. /I Theories of emotion (Vol. 1). New York: Academic. 3-33.

Prades i Artigues, L. (2008). Els brigadistes de la Guerra Civil espanyola en les fonts documentals: un prototipus de sistema d'informació digital. Tesis doctoral. Barcelona: Universidad de Barcelona, Departamento de Historia Contemporánea (2008).

Savolainen, R. (1995). Every Life Information Seeking. /I Fisher, K. E.; Erdelez, S.; McKechnie, L. (eds). Theories of Information Behavior. Medford, NJ: Inf. Today Inc.

Savolainen, R .(2014). The interplay ot affective and cognitive factors in information seeking and use. Comparing Kuhlthau's and Nahl's models. // Journal of Documentation. 71:1, 175-197.

Savolainen, R (2015). Expressing emotions in information sharing: a study of online discussion about immigration. // Information Research. 20:1 (2015). http://InformationR. net/ir/20-1/paper662.html (2015-07-28)

Scherer, K. R. (2005). What are emotions? And how can they be measured?// Social Science Information. 44:4, 695-729.

Thayer, R.E. (1996). The Origin of Everyday Moods. New York: Oxford University Press.

Wildemuth, B. M. (2009). Applications of Social Research Methods to Questions in Information and Library Science. Westport, CT: Libraries Unlimited.

Enviado: 2015-08-06. Segunda versión: 2015-09-27. Aceptado: 2016-03-18. 